\title{
Type 1 Diabetes in India: The Numbers Show the Way Ahead
}

\author{
ANJU VIRMANI \\ Senior Consultant Pediatric Endocrinologist, Max, Pentamed and Rainbow Hospitals, New Delhi, India. \\ virmani.anju@gmail.com
}

$\mathrm{T}$ The incidence and prevalence of Type 1 diabetes (T1D) is suspected to be high in India, but in the absence of a nation-wide registry, it is not possible to be sure of the numbers. The Diabetes Atlas 2017 estimates that there are 128,500 children and adolescents with diabetes in India [1]. However, extrapolating from a prevalence of 0.54 per 1000 population in expatriate Indians in UK [2], this figure translates roughly to a prevalence of 700,000 in a population of 122 crores, assuming all T1D patients are diagnosed and all survive. Where are these children disappearing? It has indeed long been suspected that many children were dying before, or soon after diagnosis [3], reducing prevalence sharply. However, as the country experiences reduction in poverty, better communication and technology, greater awareness among health care personnel (HCP), and more trained specialists, this situation appears to be improving, with earlier diagnosis and increased survival.

These children and their families need a lifetime of intensive diabetes education, multiple daily insulin injections, daily blood glucose monitoring, prevention and handling of acute complications, screening for and managing chronic complications, safe disposal of sharps, psychological support, and societal support rather than discrimination. This is thus a complex, expensive, exhausting disorder for a child and family to cope with. Unless timely and ongoing care is provided by HCP familiar with its management, the quality of life can be abysmal, worsened by the frequent chronic complications, with all their costs in turn.

Given this enormous burden of controlling diabetes as yet we cannot prevent or cure it - much greater attention and resources are needed by the HCP community, administrators, and industry. Data are urgently needed for effective planning. Some large centers have published their patient data. In 2004, Bhatia, et al. [4] reported that out of 160 diabetics (onset $<18 \mathrm{y}$ ), $81 \%$ had T1D, $8 \%$ type 2 diabetes (T2D), and 9\% fibrocalculous pancreatic diabetes mellitus. The mean $\mathrm{HbAlc}$ was $8 \%$. Ketoacidosis and severe hypoglycemia were 5.0 and 3.3 episodes per 100 patient-years. With mean (SD) disease duration of $10.2(4.6) \mathrm{y}$, retinopathy occurred in $22 \%$ and nephropathy in $18 \%$. Mortality was high at $7 \%$ over 823 patient-years of follow up.

In 617 T1D patients (onset $<20 \mathrm{y}$ ), Ramachandran, et al. [5] reported retinopathy in $13.4 \%$, nephropathy in $7.1 \%$, sensory neuropathy in $3 \%$, ischemic heart disease in $0.5 \%$ and peripheral vascular disease in $0.5 \%$. Recently, Jevalikar, et al. [6], in a series of 577 patients (onset $<25$ y: part of Youth Onset Diabetes, YDR), reported that $87.4 \%$ of T1D and $8.5 \%$ of T2D had onset before 18 years of age. The disease duration was more than two years in only $211(36.6 \%)$ patients, again suggesting very high attrition rates. Retinopathy developed in $10.1 \%$, nephropathy in $13.4 \%$ and neuropathy in $6.2 \%$, at median durations of $18 \mathrm{y}, 10.5 \mathrm{y}$ and $15 \mathrm{y}$, respectively. Moreover, $11.6 \%$ of T1D were hypothyroid, and 9.7\% had celiac disease. In a recent series of children with diabetic ketoacidosis, high rates of acute kidney injury and mortality were reported [7].

In 2006, the Indian Council of Medical Research (ICMR) set up a Registry of people with Youth Onset Diabetes (onset $<25 y$; ICMR-YDR), initially aiming to enroll all youth onset cases residing within the geographical areas of eight collaborating centers across India. Very quickly it was realized that this would not lend to a nation-wide registry or even complete ascertainment, and thus the primary objective became "to understand disease patterns or types of youth onset diabetes, including their geographical variations within the country and to estimate the burden of diabetes complications." Some salient findings [8] on 5546 patients enrolled in the first phase, till July 2011, were as follows: $63.9 \%$ had T1D and 25.3\% had T2D; while T1D predominated in very young children, T2D was substantial in Southern and Eastern India (Chennai and Dibrugarh centers); at registration, $15.4 \%$ (11.1\% of T1D; $26.4 \%$ T2D) already had at least one chronic complication of diabetes (retinopathy, nephropathy, neuropathy, coronary disease); $14.1 \%$ had at least one co-morbid condition (hypothyroidism, dyslipidemia, hypertension, tuber- 
culosis, sepsis); and $56.1 \%$ had at least one episode of hospitalization due to acute medical diabetes related conditions - ketoacidosis /uncontrolled hyperglycemia $(31.8 \%)$ and hypoglycemia (11\%).

An update of the data from Lucknow, on 512 T1D patients with onset before 18 years during January 1991 to June 2015, is reported in this issue of Indian Pediatrics [9]. Their observations partly reflect the changing face of T1D: higher proportion with onset before 5 years of age, $97 \%$ patients on multiple daily injections (MDI) regimen, almost half on analog basal insulin, and lower rates of retinopathy. In other respects, things seem unchanged. The mean $\mathrm{HbA} 1 \mathrm{C}$ was $8.3 \%$. Better glycemic control was associated with higher formal education and regular follow-up. Severe hypoglycemia remained at 3.9 episodes per 100 patient-years, especially in those less than 10 years of age. Hypertension was present in $11.7 \%$, microalbuminuria in $10.3 \%$, and gross albuminuria in 3\%. Mortality rate was high, at 1.1 per 100 patient-years over 2549 patient-years of follow-up, median diabetes duration of 102 months, with 10 of 16 deaths directly related to diabetes ( 6 due to chronic renal failure caused by diabetic nephropathy). Two patients committed suicide in the third decade of life.

What lessons can we draw? Glycemic control leaves much scope for improvement even in tertiary-care centers with multidisciplinary teams. Severe hypoglycemia occurs frequently, especially in young children. Hypertension occurs often and early - this matters, as it contributes to nephropathy, renal failure, and mortality. Death occurs often and early, with diabetes a major contributor. On the positive side, MDI is possible whatever the age, education, socio-economic status, or residence (urban or rural). Hypertension is easy to detect, and treatable. Hypoglycemia rates can be brought down with technology. The pessimists may see the glass half empty, but the optimists can see the droplets gathering into it and the level slowly rising.

Funding: None; Conflict of interest: None stated.

\section{REFERENCES}

1. International Diabetes Federation. IDF Diabetes Atlas, 8 th $e d n$. Brussels, Belgium: International Diabetes Federation, 2017. Available from: http://www.diabetesatlas.org. Accessed February 17, 2019.

2. Samanta A, Burden AC, Jones GR, Woollands IG, Clarke M, Swift PG, et al. Prevalence of insulin-dependent diabetes mellitus in Asian children. Diabet Med. 1987;4:65-7.

3. Virmani A, Ushabala P, Rao PV. Diabetes mortality in a tertiary referral hospital in India. Lancet. 1990;335:1341.

4. Bhatia V, Arya V, Dabadghao P, Balasubramanian K, Sharma K, Verghese N, et al. Etiology and outcome of childhood and adolescent diabetes mellitus in North India. J Pediatr Endocrinol Metab. 2004;17:993-9.

5. Ramachandran A, Snehalatha C, Sasikala R, Satyavani K, Vijay V. Vascular complications in young Asian Indian patients with type 1 diabetes mellitus. Diabetes Res Clin Pract. 2000;48:51-6.

6. Jevalikar G, Kohli C, Bansal B, Mishra SK, Wasir JS, Singh S, et al. Childhood and youth onset diabetes: a single center experience. Indian J Pediatr. 2016; 83:792-8.

7. Baalaaji M, Jayashree M, Nallasamy K, Singhi S, Bansal A. Predictors and outcome of acute kidney injury in children with diabetic ketoacidosis. Indian Pediatr. 2018;55:311-4.

8. The Indian Council of Medical Research (ICMR), India. Executive Summary: The Indian Council of Medical Research Registry of People with Diabetes with Young Age at Onset (ICMR-YDR), Phase 1 (2006-2011) Report. Available from: http://www.icmr.nic.in/sites/default/files/ reports/Executive\%20summary. Accessed February 21, 2019.

9. Sudhanshu S, Nair VV, Godbole T, Bhaskar Reddy SV, Bhatia E, Dabadghao P, et al. Glycemic control and longterm complications in pediatric onset type 1 diabetes mellitus: A single-center experience from Northern India. Indian Pediatr. 2019;56:191-5. 